Published in final edited form as:

Cancer Control. 2012 October ; 19(4): 309-316.

\title{
Lessons learned from Avastin
}

Joanne Mortimer, M.D., F.A.C.P,

Professor, Department of Medical Oncology \& Experimental Therapeutics, City of Hope

Comprehensive Cancer Center, 1500 East Duarte Road, Duarte, CA 91010

Helene Zonder, MN, AOCNP, NP-C, and

City of Hope Comprehensive Cancer Center, 1500 East Duarte Road, Duarte, CA 91010

Sumanta K. Pal, MD*

Assistant Professor, Department of Medical Oncology \& Experimental Therapeutics, City of Hope

Comprehensive Cancer Center, 1500 East Duarte Road, Duarte, CA 91010

\section{Abstract}

Bevacizumab has been approved in the management of metastatic colorectal cancer, non-small cell lung cancer, renal cancers, and recurrent glioblastoma multiforme. The drug received initially received accelerated approval for the treatment of advanced breast cancer. However, lack of confirmatory data from additional clinical trials resulted in loss of that indication. Both the expected and unexpected toxicities reported from clinical trials using bevacizumab have helped us both to understand the drug's mechanism of action and to identify which patients are most likely to benefit from this important agent. A biomarker that helps predict for clinical benefit is needed in order to maximize clinical benefit.

\section{Keywords}

Lessons from bevacizumab

\section{Introduction}

Bevacizumab is an important agent in the oncologic armamentarium and is currently FDAapproved for the treatment of a number of solid tumors. Despite its acknowledged benefits to patients with a number of different solid tumors, the oncologic community has focused on the recent decision by the FDA to remove treatment of advanced breast cancer from its labeling. We have learned a great deal from the drug development of bevacizumab in oncology - the approval process, its impact on underserved diseases, and the identification and management of unusual and unexpected toxicities.

Corresponding Author: Joanne Mortimer, M.D., F.A.C.P. Professor, Department of Medical Oncology \& Experimental Therapeutics, City of Hope Comprehensive Cancer Center, Duarte, CA, Office: (626) 256-4673, jmortimer@ coh.org. 


\section{Summary of the Clinical Data}

Because the growth and spread of cancers is dependent on the development of a neovasculature, angiogenesis is an important therapeutic target in oncology. Bevacizumab is a humanized antibody that targets vascular endothelial growth factor (VEGF) which stimulates blood vessel formation. Bevacizumab is not a cytotoxic agent. Its mechanism of action is believed to result from its effects on equalizing interstitial pressures within the tumor vasculature that result in improved delivery of chemotherapy. ${ }^{1-2}$ Bevacizumab has been studied in a number of solid tumors and is FDA approved in the treatment of advanced colorectal cancer, non-small cell lung cancer, advanced renal cancer and recurrent glioblastoma multiforme. Data from the phase III trials that lead to FDA approval are provided in Table 1.

\section{Colorectal Cancer}

Bevacizumab was first approved in combination with Irninotecan, 5-Fluorouracil and Leucovorin (IFL) as first line therapy for metastatic colorectal cancer which resulted in both improvement in progression free and overall survivals. Grade 3-4 adverse events were 10\% higher in the experimental arm. Six patients receiving bevacizumab experience gastrointestinal (GI) perforation, which was fatal in one patient. ${ }^{3}$ Two years later bevacizumab was approved for second line therapy in combination with 5-Fluorouracil and Leucovorin and Oxaliplatin (FOLFOX4). Even as second-line therapy progression-free (PFS) and overall survivals were improved with the addition of bevacizumab. The difference in grade 3-4 toxicities was $25 \%$ higher in the bevacizumab arm and an increased risk for bleeding became apparent. ${ }^{4}$ The benefits of bevacizumab in metastatic colorectal cancer clearly outweighed the side effects of hypertension, proteinuria, and bleeding.

\section{Lung Cancer}

Results from a phase II randomized trial of carboplatin and paclitaxel with and without bevacizumab in patients with locally advanced or metastatic non-small cell lung cancer identified bleeding in the as a potential toxicity associated with bevacizumab. Six patients whose tumors were located near major vessels, with squamous cell histology, tumor necrosis and cavitation experienced severe hemoptysis which was fatal in 4 paitents. ${ }^{5}$ Therefore, in designing the confirmatory Phase III trial, these patients were excluded from study participation as were patients with brain metastasis. The addition of bevacizumab to paclitaxel and carboplatin was superior to chemotherapy and resulted in improvements in response rate, PFS, and overall survival. Even with these exclusions, life-threatening hemoptysis was reported in $1.9 \%$ of patients and was fatal in $1.2 \% .^{6}$ In clinical practice, bevacizumab continues to be contraindicated in patients with squamous cell tumors.

\section{Breast Cancer}

Given the success of bevacizumab in two of the most common solid tumors, it seemed likely that the drug would be of benefit in women with breast cancer. The first combination trial in advanced disease enrolled 462 pretreated patients to receive capecitabine alone or capecitabine in combination with bevacizumab. Although the response rate favored the combination (19.8\% vs $9.1 \%$; $\mathrm{p}=0.001)$, the primary endpoint of improved progression free 
survival was not achieved ( 4.86 v 4.17 months; hazard ratio $=0.98) .{ }^{7}$ The higher response rate suggested a potential benefit with bevacizumab and any improvements in survival may have been masked by the fact that the patients enrolled had been heavily pretreated. The E2100 study enrolled women with newly metastatic non-HER2 positive cancers to receive either paclitaxel every 3 weeks or paclitaxel in combination with bevacizumab. No improvement in overall survival was seen, a target established by the FDA for approval of first-line therapy in this setting. However, both a higher response rate and prolongation in PFS lead to the accelerated approval of bevacizumab in this setting. ${ }^{8}$ Additional supporting data is required by the FDA in order to obtain full approval status. However, subsequent clinical trials of bevacizumab in metastatic breast cancer failed to support the encouraging data from E2100. This resulted in two additional FDA reviews and ultimately removal of a breast cancer indication for bevacizumab. The controlled trails that addressed bevacizumab as first line therapy in advanced breast cancer are summarized in Table 2 .

The AVADO trial was a three arm trial comparing docetaxel as a single agent to docetaxel with bevacizumab at two different dose levels $7.5 \mathrm{mg} / \mathrm{kg}$ and $15 \mathrm{mg} / \mathrm{kg}$. All drugs were administered at 21 days intervals. Results for the two bevacizumab arms are virtually superimposable. A significant difference of slightly over 1 month was observed in PFS favoring the bevacizumab arms but no difference was observed in overall survival. Gastrointestinal perforations were reported in $3 \%$ of patients on bevacizumab and $0.8 \%$ of patients died of bevacizumab-associated toxicity. ${ }^{9}$

The third trial, RIBBON 1, randomized patients to receive or not receive bevacizumab with whatever first line chemotherapy regimen was favored by the treating medical oncologist. The data were analyzed in two distinct chemotherapy groups by either "anthracyclinebased" or "taxane based" chemotherapy with or without bevacizumab. A significant improvement in both response rate and progression free survival was reported when bevacizumab was added to each chemotherapy regimen. However, overall survival was not improved. Of note, one in four patients discontinued bevacizumab due to toxicity and death was attributed to toxicity in $1.2 \%{ }^{10}$

\section{Renal Cell}

Two phase III studies evaluated the efficacy of bevacizumab in metastatic renal cell carcinoma (mRCC). In the AVOREN study, 649 patients were randomized to receive either bevacizumab with interferon-alpha (IFN- $a$ ) or placebo with IFN- $a$. The study satisfied its primary endpoint, demonstrating an improvement in PFS with bevacizumab therapy (10.2 vs 5.4 months; $\mathrm{HR}=0.63, \mathrm{P}<0.0001) .{ }^{11} \mathrm{~A}$ more recent update of OS in this study suggested a non-statistically significant advantage with bevacizumab, as well (23.3 vs 21.3 months; $\mathrm{HR}=0.86, \mathrm{P}=0.13) .{ }^{12}$ A second study, CALG 90206, employed a similar randomization and yielded a similar result. In CALGB 90206, 732 patients were randomized to receive either bevacizumab with IFN- $a$ or IFN- $a$ alone. In this open label study, bevacizumab was once again associated with a significant benefit in PFS (8.4 vs 4.9 months; $\mathrm{P}<0.0001$ ), and a nonsignificant improvement in OS (18.3 vs 17.4 months, $\mathrm{P}=0.097) .{ }^{13-14}$ No new or unanticipated toxicities were observed with this combination. ${ }^{15}$ 


\section{Glioblastoma}

Vascular proliferation and tumor necrosis have been a hallmark of glioblastoma multiforme and VEGF is highly expressed in these tumors. ${ }^{2,16}$ A phase II trial of bevacizumab in combination with irinotecan conducted in patients with recurrent glioblastoma multiforme resulted in a 6 month PFS of $46 \%$ and a overall survival of $57 \% .{ }^{17}$ To confirm these optimistic results a randomized trial was performed comparing the combination of bevacizumab and irinotecan to bevacizumab alone. The study assumed that patients treated with single agent irinotecan, the 6 months PFS would be $15 \%$. The 6 month PFS with single agent bevacizuamab was $42.6 \%$ and $50.3 \%$ for combination arm. Intracranial hemorrhage occurred in 4 patients, 2 developed cranial would dehiscence and 2 patients experienced GI perforations. ${ }^{18}$ Bevacizumab was approved as a single agent in patients with recurrent glioblastoma multiforme. This is the only malignancy for which bevacizumab is recommended without the co-administration of another agent. Given the unmet need, bevacizumab is an important addition to the treatment of these patients.

\section{Ovarian Cancer}

Recently the results from two controlled trials of bevacizumab in ovarian cancer have been published, one by the GOG and the other by the European ICON7 as summarized in Table 3. Both trials enrolled women with newly diagnosed ovarian cancer to receive 6 cycles of carboplatin (AUC 5-6) and paclitaxel with or without bevacizumab. When bevacizumab was assigned, the drug was initiated on cycle 2 in order to minimize postoperative complications. The GOG trial was a three-arm study. In the 2 study arms the bevacizumab was administered every 3 weeks with the chemotherapy during cycles $2-6$ or for a longer duration with cycles 2-6 and continuing every 3 weeks up to 22 cycles. In the ICON7 trial, bevacizumab was administered with chemotherapy for cycles $2-6$ and subsequently as a single agent up to 12 cycles. For both studies, PFS favors the addition of bevacizumab. In the GOG study, GI perforations were reported in all 3 treatment arms and the incidence was more than doubled in patients receiving bevacizumab. ${ }^{19-20}$ In an early Phase II study of bevacizumab added to paclitaxel and carboplatin five of 44 women with ovarian cancer developed bowel perforations. ${ }^{21}$ The GOG and ICON7 studies suggest that the increased incidence of GI perforation is comparable to what has been reported with bevacizumab in other primary cancers. ${ }^{22}$ Bevacizumab may be safely administered in patients with ovarian cancer. The pathophysiology for development of this complication remains unclear. A subset analysis of women with high risk disease who were enrolled in the ICON7 study identified an overall survival advantage to bevacizumab. Results from this unplanned analysis are encouraging and with additional follow-up an improvement in median survival may be seen for all patients enrolled. ${ }^{19-20}$

A number of important lessons have been learned from the data summarized above. Wellconducted Phase II studies often, but not always, provide important information to guide the design of Phase III studies. By identifying fatal hemoptysis in patients with squamous cell cancers, tumor necrosis and cavitation, and lesions located near major vessels such patients were excluded from study participation and toxicity from bevacizumab was minimized. ${ }^{5-6,23}$ In contrast toxicity data from a phase II trial of bevacizumab in ovarian cancer may have been misleading as a high incidence of GI perforation was observed. ${ }^{21}$ The 
unique pattern of metastasis throughout the peritoneum raised concerns in the oncology community that perforation was related to regression of tumor involving the bowel wall. In fact the incidence of gastrointestinal perforation determined from 12,294 patients enrolled in 17 randomized controlled trials was $0.9 \%$ and is associated with a mortality rate of $21.7 \% .^{22}$ With additional experience using bevacizumab, it is clear that GI perforation is a toxicity observed with bevacizumab use regardless of the primary tumor site or location of metastatic disease.

The initial clinical experience with bevacizumab raised concerns about administering the drug in the immediate postoperative setting. An increase in postoperative bleeding and compromised wound healing were reported. In designing trials, we frequently exclude patients with disease involving the brain because of bleeding concerns. However, the data in recurrent primary brain tumors support the benefit of bevacizumab. ${ }^{18}$ An exploratory analysis of CNS bleeding in patients with brain metastases reviewed the risk of CNS bleeding from patients enrolled on 13 randomized. Of 8,443 patients enrolled on study, occult metastases were identified in 187 participants, 91 of whom were assigned to receive bevacizumab. Three of the bevacizumab-treated patients developed grade 4 cerebral hemorrhage (3.3\%) compared to one of $96(1 \%)$ patients in the control arm. ${ }^{24}$ Consideration should be given to include select patients with tumor involving the brain.

Of course the overarching lesson is that well-design and conducted Phase III trails are the gold standard for advancing treatment. Adequately powered trials can identify treatment effects and associated toxicities.

\section{Adjuvant therapy trials}

To date, the only published adjuvant trial was conducted by the NSABP in colorectal cancer. In NSABP C08, 2672 patients with stage II and III colorectal cancer were randomized to receive FOLFOX6 for 26 weeks with or without 52 weeks of bevacizumab. With 3 years of median follow-up, no advantage in disease free or overall survival has been observed. ${ }^{25}$ Agents with demonstrated activity in advanced disease have generally resulted in an improvement in overall survival when used in the adjuvant setting in patients with a high risk of disease recurrence who have undergone definitive treatment of the primary tumor. The first indication for bevacizumab was for the first-line treatment of metastatic colorectal cancer and is therefore puzzling that an adjuvant trial in this disease would be negative. Is it possible that a VEGF inhibitor may not have the same impact on micrometastatic disease? A number of adjuvant trials are on-going in a variety of solid tumors and we await these results with interest.

\section{Side Effects}

The most commonly recognized toxicities observed with bevacizumab such as proteinuria, hemorrhage and thrombosis have been have been attributed to its vascular targeting activity. New onset of hypertension requiring treatment develops in up to one quarter of patients treated with bevacizumab. ${ }^{26}$ The relationship between drug toxicity and efficacy has been established as had been observed with myelosuppression with the use of adjuvant chemotherapy of breast cancer, vasomotor symptoms with endocrine therapy, and skin rash 
with the tyrosine kinase inhibitors. Although there has not been a consistent definition for "hypertension" there is a suggestion of a relationship between the new onset of hypertension and bevacizumab efficacy has been demonstrated in pivotal trials in NSCLC and renal cancers. $^{27}$

As is true with most drugs, additional side effects become apparent only after the agent has been approved and clinical experience has been gained. It is apparent that some of the toxicities of bevacizumab are more disease specific that others. We have summarized the data below:

\section{Arterial thrombosis}

In the majority of the trials reviewed, thromboembolic events were reported as side effects of treatment. Thromboembolic complications are frequently seen in patients with cancer for a variety of reasons including advanced age, the hypercoagulable state associate with cancer and inflammation, and indwelling devices that affect the vessel wall and predispose to clot formation. An increase in arterial thromboembolic complications such as myocardial and cerebrovascular events, has been observed with bevacizumab. ${ }^{28}$ However, venous thromboembolic complications are not increased. In a review of 10 randomized trials of bevacizumab in over 6,000 patients with different primary tumor types, venous thromboembolic events were not increased. ${ }^{29}$

\section{Hemorrhage}

It is postulated that the increase in hemorrhage observed with bevacizumab is due to the inability of endothelial cells to renew. ${ }^{30}$ While, the highest rates of bleeding were reported in non-small cell lung cancer, hemorrhage is a consistent toxicity observed with every disease site.

\section{Cardiac disease}

Cardiac complications associated with bevacizumab have been described in women with advanced breast cancer. In a literature review of 3,784 women enrolled in randomized trials congestive heart failure was reported in $1.6 \%$ of bevacizumab-treated patients compared to $0.4 \%$ of control patients. This represents a 4.74 risk for developing left ventricular dysfunction with bevacizumab compared to chemotherapy alone. ${ }^{31}$ In the anthracycline + bevacizumab arm of the RIBBON1, 10 cardiac events were identified in the 210 patients -8 patients developed left ventricular dysfunction and 2 patients experienced myocardial infarction. Most of the women enrolled in first-line chemotherapy trials developed recurrent disease after treatment for early stage breast cancer and it is likely that many received anthracycline-based adjuvant chemotherapy. Since left ventricular dysfunction was not reported in the colorectal, lung, renal, ovarian, or glioblastoma trials it raises the possibility that prior anthracycline therapy may contribute to the development of LV dysfunction.

\section{Ovarian function}

In an adjuvant colorectal trial of FOLFOX 6 with and without bevacizumab, ovarian failure was identified in 32/95 (34\%) women in the bevacizumab arm compared to 2 or $83(2 \%)$ in 
the chemotherapy alone arm. With cessation of therapy, 22\% have had recovery of ovarian function. This same trial identified osteonecrosis of the jaw as an additional side effect.

\section{Fatal adverse events}

Ranpura reviewed data from 16 randomized clinical trails of bevacizumab that enrolled 10,217 patients and identified a $2.5 \%$ risk of fatal adverse events. Hemorrhage was most common cause of fatality in $23.5 \%$ of cases, neutropenia in $12.2 \%$ and GI perforations in $7.1 \% .{ }^{32}$ With continued experience with bevacizumab, the incidence of fatal events is likely lower, especially with the appreciation of a subset of patients with lung cancer are at high risk of bleeding.

\section{Conclusions}

In drug development, a thorough toxicity assessment is critical for a number of reasons. Toxicity helps to guide drug dose, schedule, and feasibility. When juxtaposed with efficacy endpoints of treatment the risk: benefit determine the over value of therapy to the patient. In the case of bevacizumab as first line therapy in breast cancer, the risk benefit ratio was too great to recommend bevacizumab as standard of care. Additionally, side effects of treatment provide important information about mechanism of action and efficacy. Bleeding is a recognized complication of bevacizumab therapy, however, the most dramatic bleeding reported was in patients with non-small cell lung cancer of squamous cell histology, cavitary lesions, and tumors located near large vessels. The specificity of this toxicity may eventually help to inform us about biologic mechanisms. As we gain a better understanding of the mechanisms of the unique toxicities associated with bevacizumab, it is likely that we will identify interventions to minimize their occurrence.

In the adjuvant colorectal trial, both ovarian failure and osteonecrosis of the jaw were identified as side effects of bevacizumab. If bevacizumab is incorporated into the treatment of patients with curable diseases, it is likely that additional side effects will be identified. The aromatase inhibitors were first approved in the management of metastatic breast cancer and it was not until they were used in the adjuvant setting that arthralgias and myalgias were appreciated as a common side effect of this class of agents. The incidence of this side effect may be as high as $50 \%$ but it was impossible to attribute arthralgias and myalgias to drug in a population of women with metastatic disease. It seems likely that additional toxicities from bevacizumab will continue to be identified.

To date, there has been no pathologic or clinical marker that can predict which patients will benefit from bevacizumab. The new onset of hypertension was found to be predictive of improved outcome in some but not all trials and data from trials in breast, colorectal, and ovarian cancers, suggest that polymorphisms in the VEGF may identify a population of patients who are likely to benefit from bevacizumab. ${ }^{27}$ As we continue to "personalize" cancer care, it will be important to identify a marker for benefit from bevacizumab.

Non cytotoxic agents such as the tyrosine kinase inhibitors, trastuzumab, and bevacizumab have altered the way we think of drug development since they have no significant single agent activity and require administration with other agents to maximize their benefit. It is 
clear that bevacizumab is an important agent in the management of many advanced cancers. We are continuing to learn how best to incorporate it into the treatment of solid tumors.

\section{Acknowledgments}

Dr. Pal's efforts are supported by the NIH Loan Repayment Plan (LRP), the CBCRP 15IB-0140 (California Breast Cancer Research Program Junior IDEA Award) and NIH K12 2K12CA001727-16A1.

\section{References}

1. Willett CG, Boucher Y, di Tomaso E, et al. Direct evidence that the VEGF-specific antibody bevacizumab has antivascular effects in human rectal cancer. Nat Med. 2004; 10:145-7. [PubMed: 14745444]

2. Jain RK. Normalizing tumor vasculature with anti-angiogenic therapy: A new paradigm for combination therapy. Nat Med. 2001; 7:987-9. [PubMed: 11533692]

3. Hurwitz H, Fehrenbacher L, Novotny W, et al. Bevacizumab plus Irinotecan, Fluorouracil, and Leucovorin for Metastatic Colorectal Cancer. New England Journal of Medicine. 2004; 350:233542. [PubMed: 15175435]

4. Giantonio BJ, Catalano PJ, Meropol NJ, et al. Bevacizumab in Combination With Oxaliplatin, Fluorouracil, and Leucovorin (FOLFOX4) for Previously Treated Metastatic Colorectal Cancer: Results From the Eastern Cooperative Oncology Group Study E3200. Journal of Clinical Oncology. 2007; 25:1539-44. [PubMed: 17442997]

5. Johnson DH, Fehrenbacher L, Novotny WF, et al. Randomized Phase II Trial Comparing Bevacizumab Plus Carboplatin and Paclitaxel With Carboplatin and Paclitaxel Alone in Previously Untreated Locally Advanced or Metastatic Non-Small-Cell Lung Cancer. Journal of Clinical Oncology. 2004; 22:2184-91. [PubMed: 15169807]

6. Sandler A, Gray R, Perry MC, et al. Paclitaxel-Carboplatin Alone or with Bevacizumab for NonSmall-Cell Lung Cancer. New England Journal of Medicine. 2006; 355:2542-50. [PubMed: 17167137]

7. Miller KD, Chap LI, Holmes FA, et al. Randomized Phase III Trial of Capecitabine Compared With Bevacizumab Plus Capecitabine in Patients With Previously Treated Metastatic Breast Cancer. Journal of Clinical Oncology. 2005; 23:792-9. [PubMed: 15681523]

8. Miller K, Wang M, Gralow J, et al. Paclitaxel plus Bevacizumab versus Paclitaxel Alone for Metastatic Breast Cancer. New England Journal of Medicine. 2007; 357:2666-76. [PubMed: 18160686]

9. Miles DW, Chan A, Dirix LY, et al. Phase III Study of Bevacizumab Plus Docetaxel Compared With Placebo Plus Docetaxel for the First-Line Treatment of Human Epidermal Growth Factor Receptor 2-Negative Metastatic Breast Cancer. Journal of Clinical Oncology. 2010; 28:3239-47. [PubMed: 20498403]

10. Robert NJ, Diéras V, Glaspy J, et al. RIBBON-1: Randomized, Double-Blind, Placebo-Controlled, Phase III Trial of Chemotherapy With or Without Bevacizumab for First-Line Treatment of Human Epidermal Growth Factor Receptor 2-Negative, Locally Recurrent or Metastatic Breast Cancer. Journal of Clinical Oncology. 2011; 29:1252-60. [PubMed: 21383283]

11. Escudier B, Koralewski P, Pluzanska A, et al. A randomized, controlled, double-blind phase III study (AVOREN) of bevacizumab/interferon- $\{$ alpha $\} 2$ a vs placebo/interferon- $\{$ alpha $\} 2 \mathrm{a}$ as firstline therapy in metastatic renal cell carcinoma. ASCO Meeting Abstracts. 2007; 25:3.

12. Escudier B, Bellmunt J, Négrier S, et al. Phase III Trial of Bevacizumab Plus Interferon Alfa-2a in Patients With Metastatic Renal Cell Carcinoma (AVOREN): Final Analysis of Overall Survival. Journal of Clinical Oncology. 2010; 28:2144-50. [PubMed: 20368553]

13. Rini BI, Halabi S, Rosenberg J, et al. Bevacizumab plus interferon-alpha versus interferon-alpha monotherapy in patients with metastatic renal cell carcinoma: Results of overall survival for CALGB 90206. J Clin Oncol (Meeting Abstracts). 2009; 27 LBA5019. 
14. Rini BI, Halabi S, Rosenberg JE, et al. Phase III Trial of Bevacizumab Plus Interferon Alfa Versus Interferon Alfa Monotherapy in Patients With Metastatic Renal Cell Carcinoma: Final Results of CALGB 90206. J Clin Oncol. 2010; 28:2137-43. [PubMed: 20368558]

15. Escudier B, Cosaert J, Pisa P. Bevacizumab: direct anti-VEGF therapy in renal cell carcinoma. Expert Review of Anticancer Therapy. 2008; 8:1545-57. [PubMed: 18925847]

16. Godard S, Getz G, Delorenzi M, et al. Classification of Human Astrocytic Gliomas on the Basis of Gene Expression. Cancer Research. 2003; 63:6613-25. [PubMed: 14583454]

17. Vredenburgh JJ, Desjardins A, Herndon JE, et al. Bevacizumab Plus Irinotecan in Recurrent Glioblastoma Multiforme. Journal of Clinical Oncology. 2007; 25:4722-9. [PubMed: 17947719]

18. Friedman HS, Prados MD, Wen PY, et al. Bevacizumab Alone and in Combination With Irinotecan in Recurrent Glioblastoma. Journal of Clinical Oncology. 2009; 27:4733-40. [PubMed: 19720927]

19. Burger RA, Brady MF, Bookman MA, et al. Incorporation of Bevacizumab in the Primary Treatment of Ovarian Cancer. New England Journal of Medicine. 2011; 365:2473-83. [PubMed: 22204724]

20. Perren TJ, Swart AM, Pfisterer J, et al. A Phase 3 Trial of Bevacizumab in Ovarian Cancer. New England Journal of Medicine. 2011; 365:2484-96. [PubMed: 22204725]

21. Cannistra SA, Matulonis UA, Penson RT, et al. Phase II Study of Bevacizumab in Patients With Platinum-Resistant Ovarian Cancer or Peritoneal Serous Cancer. Journal of Clinical Oncology. 2007; 25:5180-6. [PubMed: 18024865]

22. Hapani S, Chu D, Wu S. Risk of gastrointestinal perforation in patients with cancer treated with bevacizumab: a meta-analysis. The Lancet Oncology. 2009; 10:559-68. [PubMed: 19482548]

23. Ramalingam SS, Dahlberg SE, Langer CJ, et al. Outcomes for Elderly, Advanced-Stage Non Small-Cell Lung Cancer Patients Treated With Bevacizumab in Combination With Carboplatin and Paclitaxel: Analysis of Eastern Cooperative Oncology Group Trial 4599. J Clin Oncol. 2008; 26:60-5. [PubMed: 18165641]

24. Besse B, Lasserre SF, Compton P, Huang J, Augustus S, Rohr U-P. Bevacizumab Safety in Patients with Central Nervous System Metastases. Clinical Cancer Research. 2010; 16:269-78. [PubMed: 20028762]

25. Allegra CJ, Yothers G, O'Connell MJ, et al. Phase III Trial Assessing Bevacizumab in Stages II and III Carcinoma of the Colon: Results of NSABP Protocol C-08. Journal of Clinical Oncology. 2011; 29:11-6. [PubMed: 20940184]

26. Maitland ML, Bakris GL, Black HR, et al. Initial Assessment, Surveillance, and Management of Blood Pressure in Patients Receiving Vascular Endothelial Growth Factor Signaling Pathway Inhibitors. Journal of the National Cancer Institute. 2010; 102:596-604. [PubMed: 20351338]

27. Jubb AM, Harris AL. Biomarkers to predict the clinical efficacy of bevacizumab in cancer. The Lancet Oncology. 2010; 11:1172-83. [PubMed: 21126687]

28. Stone RL, Sood AK, Coleman RL. Collateral damage: toxic effects of targeted antiangiogenic therapies in ovarian cancer. The Lancet Oncology. 2010; 11:465-75. [PubMed: 20226736]

29. Hurwitz HI, Saltz LB, Van Cutsem E, et al. Venous Thromboembolic Events With Chemotherapy Plus Bevacizumab: A Pooled Analysis of Patients in Randomized Phase II and III Studies. Journal of Clinical Oncology. 2011; 29:1757-64. [PubMed: 21422411]

30. Kilickap S, Abali H, Celik I. Bevacizumab, Bleeding, Thrombosis, and Warfarin. Journal of Clinical Oncology. 2003; 21:3542. [PubMed: 12972536]

31. Choueiri TK, Mayer EL, Je Y, et al. Congestive Heart Failure Risk in Patients With Breast Cancer Treated With Bevacizumab. Journal of Clinical Oncology. 2011; 29:632-8. [PubMed: 21205755]

32. Ranpura V, Hapani S, Wu S. Treatment-Related Mortality With Bevacizumab in Cancer Patients. JAMA: The Journal of the American Medical Association. 2011; 305:487-94. [PubMed: 21285426] 


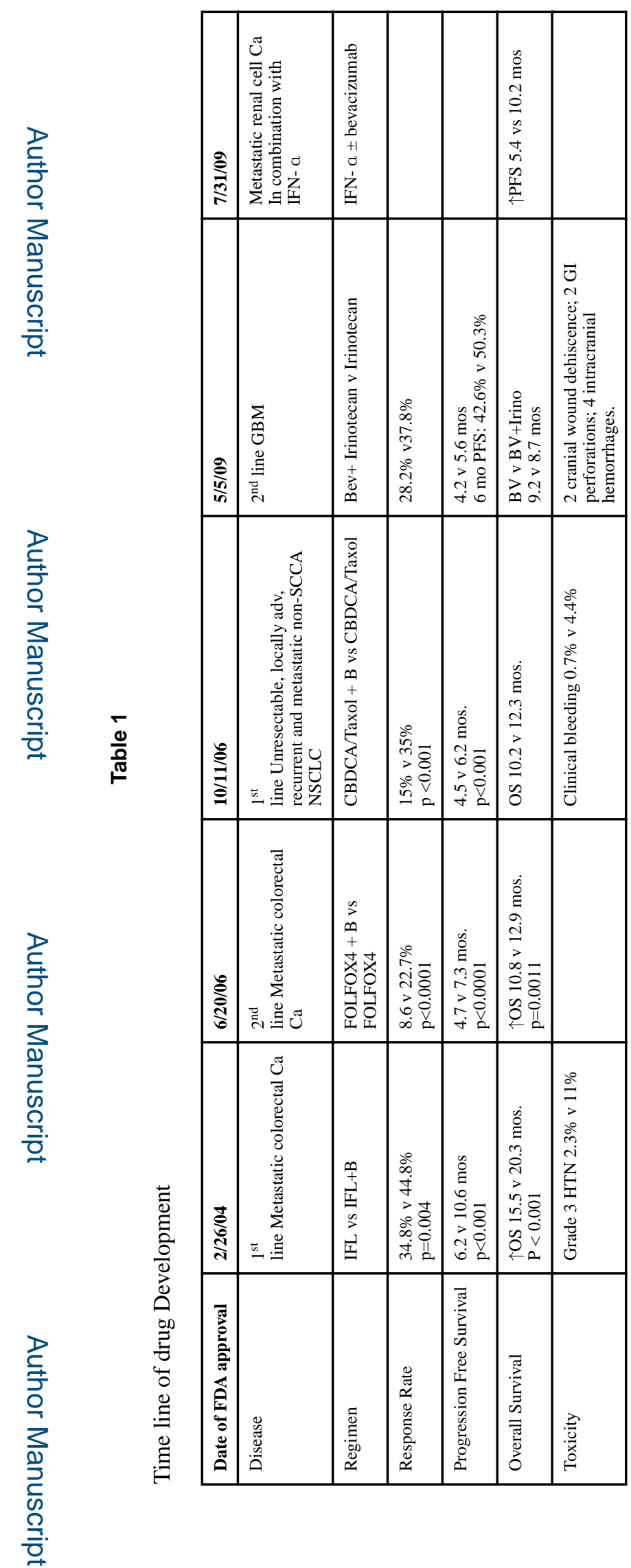

Cancer Control. Author manuscript; available in PMC 2016 August 01. 


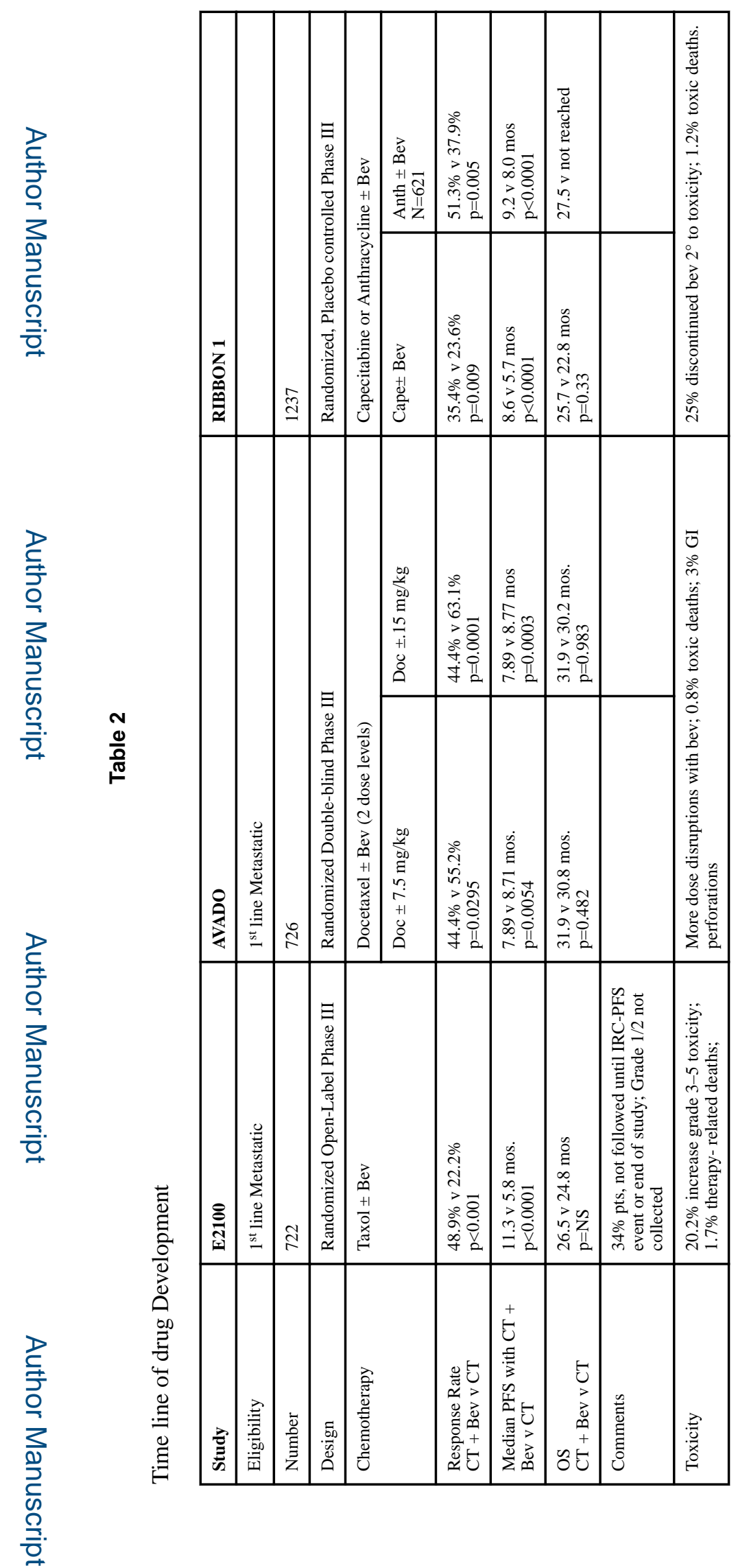

Cancer Control. Author manuscript; available in PMC 2016 August 01. 


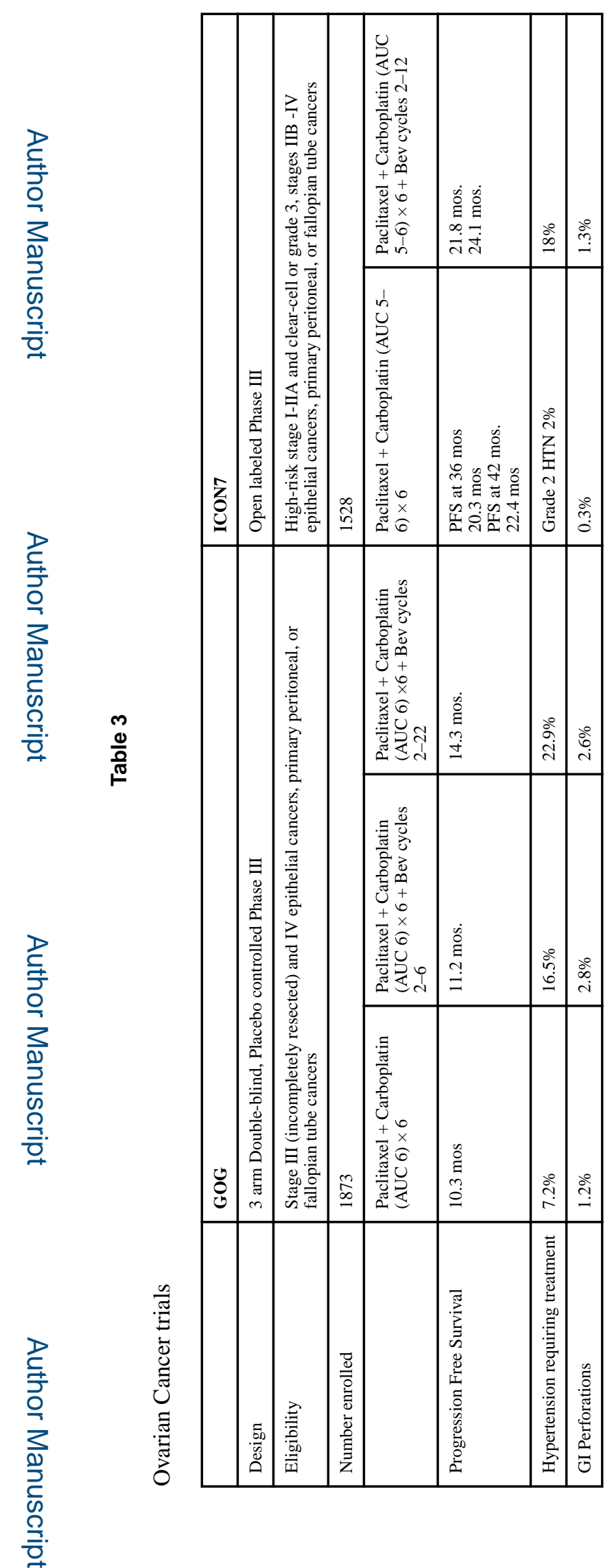

Cancer Control. Author manuscript; available in PMC 2016 August 01. 